\title{
Precision Medicine and Site-Specific Karel Petrak Drug Delivery
}

University of Sussex, London, England

\section{Abstract}

Precision medicine is an approach to prevent and treat diseases that takes into account people's individual variations in genes, environment, and lifestyle. The current Precision Medicine Initiative of the US Government is to "generate the scientific evidence needed to move the concept of precision medicine into clinical practice". In the first approximation, precision medicine may provide a more accurate diagnosis of the disease such as cancer, but may not have the means to offer an improved therapy.

The aim of drug targeting is to generate pharmacologically effective drug concentration at the site of disease while keeping a very low/minimal drug concentration in the rest of the body, away from the site of disease. Targeted drugs are thus "precision drugs" needed to bring precision medicine into the clinical practice.

Focusing on the area of cancer therapy, this review examines the essential requirements that must be met for cell/tumour-targeted drug-delivery systems to work. It examines the progress to date and draws conclusions for an optimal paradigm for future drug-delivery systems development.

\section{Corresponding author: Karel Petrak}

\section{” klpetrak@gmail.com}

D.Phil., Studies in Cancer, Independent Consultant, Houston, Texas, USA

Tel: +541147747472

\section{Introduction}

Precision Medicine is a medical-research area that recently attracted headline attention when President Obama announced a \$US215 million initiative to amass genetic data on some 1 million Americans, with the aim of "discovering genetic causes of disease and finding new drugs that will target dangerous mutations" [1].

The aim of precision medicine is to take into account people's individual variations in genes, environment, and lifestyle when treating diseases. Accordingly, the current precision-medicine initiative of the US Government is to "generate the scientific evidence needed to move the concept of precision medicine into clinical practice" $[2,3]$.

The current use of antibiotics offers a simple illustration of precision-medicine application. When presented with symptoms of bacterial infection, physicians prescribe an antibiotic. Precision-medicine approach would be rapidly to determine what bacterium is involved and then prescribe the most appropriate antibiotic.

Near-term goals of Precision Medicine include focusing on cancer [4]. Accordingly innovative clinical trials of targeted drugs for adult are to be carried out.
In this context, the term "targeted drugs" can have at least two interpretations

1. Drugs that act on, "target", a specific biological/disease molecular mechanism; such drugs often act on a range of cells and not exclusively at the site of disease and as such will exert not only the desired therapeutic effects but also other, sometime even dose-limiting toxic effects.

2. Drugs that are delivered to specific anatomical area or organ/tissue or ideally and exclusively to specific cells that are the focus of the disease to be treated. The issue here is that there are currently no clinically effective targeted "precision drugs" available.

In the first approximation, precision medicine may provide a more accurate diagnosis of the disease such as cancer, but may not have the means to offer an improved therapy. Targeting cells in which a precisely diagnosed mechanism drives disease such as for example any particular cancer will very likely need "precision drugs" / "precision medications" that are yet to be developed.

The concept of "precision drugs" was prophesized by Paul Ehrlich in 1906, predicting that chemists "would soon be able to produce substances that would seek out specific disease-causing agents" [5]. He used the term "magic bullets." 
Very few drugs have been developed so far to act as "magic bullets", with a notable exception of antibodies having therapeutic effect $[6,7]$. Instead, considerable efforts have been made over the last few decades to deliver existing drugs to specific disease targets $[8,9]$. The aim of this "drug targeting' has been, using drugs that can generally freely distribute throughout the body, to generate pharmacologically effective drug concentration at the site of disease while keeping a very low/minimal drug concentration in the rest of the body, away from the site of disease. These efforts have been largely unsuccessful [8-10].

In an earlier publication [11] we examined the essential requirements that must be met for cell/organ/tissue-targeted drug-delivery systems to work, and provided a mathematical definition of the conditions required for cell-specific targeting of drugs to occur. General rules derived from our analysis can be expressed verbally as follows.

1. It is essential that the drug-carrier conjugate is not removed too rapidly from circulation. The liver is mainly responsible for the removal of drug conjugates from the circulation.

2. Release of drug away from the target site could nullify any benefits that might potentially come from delivering the drug to the target site.

3. If the drug conjugate reaches the target site too slowly, the supply of free drug (as governed by its rate of release at the site from its conjugate) might never be sufficient to generate the concentration required to elicit the desired therapeutic effect at the site of action.

4. The capacity of the system selected for the release of free drug from the conjugate needs to process the entirety of the drug-carrier conjugate arriving at the target site, and doing so at a rate that ensures drug accumulation at this site.

5. Only drugs that are retained at the site of delivery and action benefit from targeting. Most existing drugs would have a tendency to diffuse away from the site when converted into their free, non-conjugated form.

6. The rate of elimination of free drug from the systemic circulation should be rapid relative to its escape from the target site.

\section{The Current Concepts of Drug-Targeting Systems are Based on Three Components}

\section{Targeting structure}

An essential prerequisite to site-specific targeting of drugs is the existence of unique molecular features, a "unique address" if you like, associated with the target of disease. Many such drug targets are potentially available [12]. The therapeutic target should be abundantly expressed by most diseased cells or tissues and absent from healthy tissues $[13,14]$. Opportunities exist for utilizing unique molecular structures for targeting to various organs and tissues, including cancer molecules and tissues. Notably, antibodies (Abs) have been raised to such unique molecular structures and used as therapeutic agents. More than 20 monoclonal antibodies have been approved to date as therapeutic drugs by the US Food and Drug Administration (for example, Alemtuzumab, Bevacizumab, Cetuximab, Gemtuzumab, Palivizumab, Panitumumab, Rituximab) [15]. The use of antibodies as carriers of other drugs to specific targets has been explored since antibodies exhibit many relevant properties - good solubility and stability, avoidance of removal from circulation by the liver, high selectivity and specificity, and bioconversion to non-toxic metabolites [16].

\section{Drug-attachment/carrying element}

Much has been published about drug carriers - a PubMed search for "drug AND carrier*" performed in August 2015 produced 44,070 hits. Rationale for the use of carriers for drugs assumes that biodistribution and pharmacokinetics of drugs can be altered by attaching to a macromolecular carrier and that the drug will consequently follow the biodistribution and the kinetics of the carrier [17]. This is, however true only until the drug is released, as it must be, from the carrier. The free drug then follows its own distribution and pharmacokinetics. In general, such drug carriers do not have any ability specifically to interact with disease targets. Instead, such carriers reach disease targets by passive distribution and hence only a very small fraction of the administered dose reaches the desired anatomical location. Although many of the carriers, for example water-soluble polymers, can remain in circulation for an extended time, attaching a meaningful amount of drug to such macromolecules significantly alters their behaviour and hence their ability to remain in circulation [18].

\section{The drug to be delivered}

It should be noted that site-targeting of conventional drugs that typically reach their targets of action via absorption across biological membranes is unlikely to be effective. The reason is that these drugs can equally well diffuse away after their release at the site. Instead, drugs selected for targeting should a) have a very high potency (i.e., a very low pharmacodynamic concentration) and should have a very low tendency to be removed (either by degradation/metabolism, binding or diffusion) from the target site after their release (i.e., in their free form). New drugs should act on the intended therapeutic target, with minimal effects on other biomolecules $[8,9,11]$.

The above overview suggests that the most plausible paradigm for the future development of site-specific drug-delivery systems is to combine monoclonal antibodies with drugs that meet the pharmacokinetic and pharmacodynamic requirements of this application. The successful use of antibodies to deliver cancer drugs is not new. Rowland et al. [19] results published in 1980s showed that "...the administration of Ida-anti-CD19 conjugates can result in complete tumour regression in an experimental model" (sic). However, translation of this technology to humans has proven to be difficult [20].

Some of the reasons that must be considered are that antibodies have very poor oral bioavailability, only a partial absorption after intramuscular or subcutaneous administration, and an uneven biological distribution and elimination. Extravasation of antibodies after systemic administration is slow as would be expected for molecules of antibody size. The rate of distribution 
in tissues is correspondingly also slow, further slowed down in tissues that possess corresponding antibody-binding epitopes. Therapeutic monoclonal antibodies (mAbs) show high affinity and extraordinary specificity for their targets. However, mAbs can only access targets on the cell surface or in the extracellular space, while most disease-specific targets reside inside of target cells. The mode of action of antibody-based drug delivery will need to engage cellule uptake (e.g., by endocytosis). Mathematical models suggest that antigen-antibody binding in tumors can retard antibody percolation and that increasing antibody dose leads to better percolation and more uniform distribution [21,22]. Further, antibodies carrying drugs may be expected to elicit an immune responses leading to the generation of endogenous antibodies against the protein. Even if a completely human Abs is used for delivering a drug, the attachment of the drug to the Abs may lead to the construct to be seen as a foreign protein, this resulting in a decrease of drug-delivery efficacy. Consequently, the type and strength of an immune response to Ab-drug constructs must be always taken into consideration.

\section{Conclusions}

Precision Medicine initiatives is likely to offer more precise diagnosis of disease, however, "precise drugs" will need to be developed for the diagnosis to be followed by equally precise therapy. Based on the current status of developing site-specific drug-delivery systems, it is concluded that research in this field need do adopt a new paradigm that centers on "self-targeting carriers" such as antibodies combined with "high-potency" drugs specifically selected or developed de novo that fully meet the specific pharmacokinetic requirements of targeted drug delivery. The mechanism of drug delivery using antibodies as carriers will need to involve not only recognition but also a cellular uptake (e.g., by endocytosis) of the antibody-drug constructs.

\section{Acknowledgement}

This work has been supported by the National Institute of Health (grant number 4R44HD057744) 


\section{References}

1 Fox JL (2015) Obama catapults patient-empowered Precision Medicine. Nat Biotechnol 33: 325.

2 http://www.nih.gov/precisionmedicine/

3 http://www.nih.gov/precisionmedicine/infographic-printable.pdf

4 http://ghr.nlm.nih.gov/handbook/precisionmedicine/initiative

5 http://www.chemheritage.org/discover/online-resources/ chemistry-in-history/themes/pharmaceuticals/preventing-andtreating-infectious-diseases/ehrlich.aspx

6 Scott AM, Wolchok JD, Old LJ (2012) Antibody therapy of cancer. Nat Rev Cancer 12: 278-287.

7 http://www.cancer.org/treatment/treatmentsandsideeffects/ treatmenttypes/immunotherapy/immunotherapy-monoclonalantibodies

8 Petrak K (2006) Nanotechnology and site-targeted drug delivery. J Biomater Sci Polym Ed 17: 1209-1219.

9 Petrak K (2011) Targeted Drug Delivery. Quo Vadis Drug Development Research 59-65.

10 Bae YH, Park K (2011) Targeted drug delivery to tumors: Myths, reality and possibility. J Control Release 153: 198-205.

11 Boddy A, Aarons L, Petrak K (1989) Efficiency of drug targeting: steady-state considerations using a three-compartment model. Pharm Res 6: 367-372.

12 Imming P, Sinning C, Meyer A (2006) Drugs, their targets and the nature and number of drug targets. Nat Rev Drug Discov 5: 821-834.
13 Segota E, Bukowski RM (2004) The promise of targeted therapy: Cancer drugs become more specific. Cleve Clin J Med 71: 551-560.

14 Weidanz JA, Hildebrand WH (2011) Expanding the targets available to therapeutic antibodies via novel disease-specific markers. Int Rev Immunol 30: 312-327.

15 Adler MJ, Dimitrov DS (2012) Therapeutic antibodies against cancer. Hematol Oncol Clin North Am 26: 447-481, vii.

16 Dolman ME (2011) Design of kidney-targeted drug-carrier conjugates for the inhibition of profibrotic signaling cascades. Ph.D. thesis, Department of Pharmaceutics, Utrecht University

17 Liechty WB, Kryscio DR, Slaughter BV, Peppas NA (2010) Polymers for drug delivery systems. Annu Rev Chem Biomol Eng 1: 149-173.

18 Uchegbu IF, Schatzlein AG (2006) Polymers in Drug Delivery. CRC Press 280.

19 Rowland AJ, Pietersz GA, McKenzie IF (1993) Preclinical investigation of the anti-tumor effects of anti-CD19-idarubicin immunoconjugates. Cancer Immunol Immunother 37: 195-202.

20 Alley SC, Okeley NM, Senter PD (2010) Antibody-drug conjugates: targeted drug delivery for cancer. Current Opinion in Chemical Biology. Next Generation Therapeutics 529-537.

21 Eger RR, Covell DG, Carrasquillo JA, Abrams PG, Foon KA, et al. (1987) Kinetic model for the biodistribution of an 111 In-labeled monoclonal antibody in humans. Cancer Res 47: 3328-3336.

22 Fujimori K, Covell DG, Fletcher JE, Weinstein JN (1990) A modeling analysis of monoclonal antibody percolation through tumors: A binding-site barrier. J Nucl Med 31: 1191-1198. 